Association for Information Systems AIS Electronic Library (AISeL)

Wirtschaftsinformatik Proceedings 2003

Wirtschaftsinformatik

September 2003

\title{
Zukunft der Arbeit - Leben und Arbeiten in einer vernetzten, mobilen Welt
}

Hans-Jörg Bullinger

Fraunhofer-Gesellschaft, München, hans-joerg.bullinger@zv.fraunhofer.de

Rolf Ilg

Fraunhofer-Institut für Arbeitswirtschaft und Organisation, Stuttgart

Follow this and additional works at: http://aisel.aisnet.org/wi2003

\section{Recommended Citation}

Bullinger, Hans-Jörg and Ilg, Rolf, "Zukunft der Arbeit - Leben und Arbeiten in einer vernetzten, mobilen Welt" (2003). Wirtschaftsinformatik Proceedings 2003. 1.

http://aisel.aisnet.org/wi2003/1

This material is brought to you by the Wirtschaftsinformatik at AIS Electronic Library (AISeL). It has been accepted for inclusion in Wirtschaftsinformatik Proceedings 2003 by an authorized administrator of AIS Electronic Library (AISeL). For more information, please contact elibrary@aisnet.org. 
In: Uhr, Wolfgang, Esswein, Werner \& Schoop, Eric (Hg.) 2003. Wirtschaftsinformatik 2003: Medien - Märkte - Mobilität, 2 Bde. Heidelberg: Physica-Verlag

ISBN: 3-7908-0111-9 (Band 1)

ISBN: 3-7908-0116-X (Band 2)

(C) Physica-Verlag Heidelberg 2003 


\title{
Zukunft der Arbeit - Leben und Arbeiten in einer vernetzten, mobilen Welt
}

\author{
Hans-Jörg Bullinger \\ Fraunhofer-Gesellschaft, München
}

\author{
Rolf Ilg \\ Fraunhofer-Institut für Arbeitswirtschaft und Organisation, Stuttgart
}

Zusammenfassung: Die weltweite Vernetzung der Unternehmen hat einen wesentlichen Einfluss auf unsere Arbeitswelt. Die Zusammenhänge sind für den Einzelnen kaum noch durchschaubar. In diesem Beitrag werden einige wesentliche Einflussparameter für die Zukunft der Arbeit beleuchtet. Konsequenzen für die Unternehmen und den Einzelnen werden aufgezeigt.

Schlüsselworte: Arbeitswelt, Vernetzung, Innovation, Zukunft der Arbeit

\section{Von der Industriegesellschaft zur Informations- und Wissensgesellschaft}

Der Wandel von der Industriegesellschaft zur Informations- und Wissensgesellschaft ist offensichtlich und beeinflusst alle Volkswirtschaften dieser Welt. Dabei sind die Internationalisierung und Globalisierung der Wirtschafts-, Arbeits- und Sozialbeziehungen die wesentlichen Auslöser und treibenden Kräfte für die zukünftigen Entwicklungen der Arbeit. Bislang eher abgeschottete oder nebeneinander existierende nationale Absatz- und Arbeitsmärkte werden in einen immer intensiveren globalen Wettbewerb eingebunden. Dabei werden nationale Schwächen und Defizite schonungslos aufgedeckt. Bei allen Schwierigkeiten, die so ein Wandel mit sich bringt, müssen jedoch auch die Chancen für neue Kooperationen und länderübergreifende Geschäftsmodelle gesehen werden.

Die globale Verfügbarkeit von Produkten, die sich in Qualität, Leistungsvermögen und Technologie angeglichen haben, zwingen die Unternehmen zu einem grundsätzlichen Überdenken ihrer Marktaktivitäten. Um sich zukünftig nachhaltige Wettbewerbsvorteile zu verschaffen, steht die Gestaltung von effizienten Geschäftsprozessen gegebenenfalls durch Fusion oder im Netzwerk mit anderen Unternehmen im Vordergrund. Durch die Privatisierung großer staatlicher Monopolisten und die damit verbundene Deregulierung ist eine große Bewegung und Un- 
ruhe in den Markt gekommen. Nun gilt es, die Unternehmensstrategien auf Flexibilität, das konsequente Management der Kundenbeziehungen sowie den Aufbau von Kompetenznetzwerken auszurichten, um damit die Turbulenzen beherrschen zu lernen. In diesem Zusammenhang lassen sich eine Reihe von Trends identifizieren, die diese Strategien beinflussen.

\subsection{Information und Wissen als Produktionsfaktor}

Wissen wird für die Unternehmen zu einem entscheidenden Faktor für Wachstum und Beschäftigungssicherung. Ableiten lässt sich das auch daran, dass neben den weniger qualifizierten Bevölkerungsgruppen auch die hoch- aber offensichtlich fehlqualifizierten Fachkräfte in den Arbeitslosenstatistiken erscheinen. Es ist also nicht ausreichend, Fach- und Expertenqualifikation aufzubauen, sondern es gilt vielmehr, die vom Markt geforderten Kompetenzen schnell und zielorientiert bereitstellen zu können. Damit wird die Fähigkeit zum flexiblen, schnellen und marktorientierten Wissensaufbau zur Schlüsselkompetenz in der Wissensgesellschaft.

Was verstehen wir unter dem Begriff der Wissensgesellschaft? Es kann vereinfachend dann von einer Wissensgesellschaft bzw. wissensbasierten Gesellschaft gesprochen werden, wenn insbesondere die wertschöpfenden Prozesse so vom Wissen einzelner Personen bzw. Unternehmen abhängen, dass die Bewirtschaftung dieses Wissens gegenüber anderen Faktoren vorrangig wird. Den Mittelpunkt gesellschaftlicher und unternehmerischer Anstrengungen bildet damit die kontinuierliche Weiterentwicklung des Wissens (Stichwort: Lifelong Learning) und dessen Anwendung.

\subsection{Informations- und Kommunikationstechniken beeinflussen die Geschäftsprozessoptimierung}

Der Einsatz neuer Informations- und Kommunikationstechniken hat heutzutage vor allem das Ziel, eine weltweite, schnelle und qualitativ hochwertige Verfügbarkeit von Informationen sicherzustellen und Geschäftsprozesse elektronisch zu unterstützen. Electronic Business und Collaborative Business stehen heute als Ausdruck für eine ganze Reihe von Aktivitäten, die mittels der Internet-Technologie zu neuen Abwicklungen geschäftlicher Transaktionen im Unternehmen und zwischen Unternehmen geführt haben und noch führen werden. Schlagworte hierzu sind E-Procurement, E-Supply Chain Management, Workflow und Data Management, E-Customer Relationship Management, E-Commerce, C-Commerce (Collaborative Commerce) und M-Commerce (Mobile Commerce).

Was neue Geschäftsmodelle anbetrifft, so prognostizieren Marktforscher im $\mathrm{Zu}$ sammenhang mit dem schneller werdenden Internet der zweiten Generation vor allem virtuellen Marktplätzen hohe Zuwachsraten. Die Vorreiterrolle übernimmt 
hier vor allem die IT-Branche, der Finanzdienstleistungssektor, die Automobilbranche sowie die Bau-, Chemie- und Energiewirtschaft. Durch die Unterstützung von bilateralen B2B-Beziehungen entlang der Wertschöpfungskette können zusätzliche Dienste, z.B. Kooperationsplattformen oder Communities, generiert werden.

\subsection{Produktionsarbeit nimmt ab - Wissensintensive Dienst- leistungen nehmen $\mathrm{zu}$}

Der Dienstleistungssektor übernimmt mehr und mehr die Rolle eines Innovationskatalysators. Herausragendes Merkmal des wirtschaftlichen und gesellschaftlichen Strukturwandels ist die wachsende Bedeutung von Dienstleistungen für den Wettbewerb und den Arbeitsmarkt: Der Dienstleistungssektor ist und bleibt ein Motor des wirtschaftlichen Wachstums und der Beschäftigungszunahme. In Deutschland wird die Produktionsarbeit noch weiter sinken, während die wissensintensiven Dienstleistungen überproportional steigen. Zahlreiche amerikanische, aber auch immer mehr europäische Unternehmen machen vor, wie systematisch und kundenorientiert entwickelte Dienstleistungen zum Schlüssel für den Erfolg werden. Dabei steht häufig nicht die alleinige Dienstleistung im Interesse der Unternehmen, sondern vielmehr der Mix eines Hardware-Produkts mit einer oder mehreren dazu passenden Dienstleistungsangeboten. Dies wird häufig als hybrides Produkt bezeichnet.

Nur wenige Unternehmen haben frühzeitig erkannt, dass angesichts der zunehmenden Globalisierung die entsprechenden Wettbewerbsvorteile in der Zukunft nicht mehr allein durch Technologievorsprünge, Kostenführerschaft oder Produktqualität erzielt werden können. Vielmehr wird gerade die Differenzierung über innovative Dienstleistungen zu einem Alleinstellungsmerkmal gegenüber Wettbewerbern und damit zu einer erfolgversprechenden Strategie zur Erschließung neuer Marktpotenziale.

In der Dienstleistungsforschung haben sich u.a. drei wichtige Bereiche herauskristallisiert. Neben dem Benchmarking zur Bewertung von Dienstleistungen und zur Identifizierung von Dienstleistungslücken sind die Bereiche Service Engineering und Standardisierung in Deutschland weiterentwickelt worden. Inzwischen existieren Methoden, Klassifizierungen, Prozess- und Marketingmodelle sowie unterstützende Standards zur Entwicklung und Implementierung neuer Dienstleistungsprodukte. Um die Chancen der Dienstleistungen besser nutzen zu können bedarf es einer weiteren Verbesserung der Wissensbasis durch die systematische Erforschung, Reflektion und Nutzung von internationalen Good Practice Beispielen und eines Mentalitätswandels durch Commitment

- in der Wirtschaft, bei Unternehmern und Mitarbeitern, die den Charakter und Wert von Dienstleistungen erkennen und im Dienst am Kunden ihre Leistung und ihre Zukunft sehen, 
- in der Politik, bei verantwortungsvollen und mutigen Entscheidern, die sich selber auch als Service-Einheiten für den Wirtschafts- und Lebensstandort Deutschland sehen und der Schaffung günstiger Rahmenbedingungen für Dienstleister und Dienstleistungen explizite und hohe Priorität einräumen,

- in der Gesellschaft, bei uns allen als Nachfrager und Koproduzenten traditioneller und innovativer Dienstleistungen.

Die Erkenntnis über den Zusammenhang von systematischer Entwicklung und Markterfolg, wie er für klassische Hardware-Produkte und in den letzten 20 Jahren für Software-Produkte gilt, wird sich zukünftig auch bei Dienstleistungsprodukten einstellen.

\subsection{Demographische Entwicklung: Menschen werden immer älter}

Die Betrachtung der demographischen Entwicklung zeigt, dass es durch sinkende Geburtenraten und einer kontinuierlichen Steigerung der Lebensdauer mittel- bis langfristig zu einer erheblichen Veränderung der Altersstrukturen in Deutschland kommt. Die Prognosen gehen von einem Bevölkerungsrückgang aus, der eine Abnahme der Zahl jüngerer Erwerbsfähiger zur Folge hat. Vor diesem Hintergrund sind vor allem Engpässe bei der Rekrutierung von qualifiziertem betrieblichen Nachwuchs und ein erhöhtes Durchschnittsalter der Belegschaften zu erwarten.

Die Unternehmen müssen heute schon Maßnahmen ergreifen und sich auf diese veränderten Rahmenbedingungen einstellen. Besonders gravierend ist in diesem Zusammenhang immer noch das weit verbreitete Vorurteil, dass ältere Menschen weniger leistungs- und innovationsfähig seien. Hierzu liegen aktuelle Forschungsergebnisse vor, die belegen, dass Leistungskraft und Innovationsfähigkeit weniger mit dem biologischen Alter(n) als vielmehr damit zusammenhängt, ob die Menschen in ihren individuellen Lebens-, Erwerbs- und Berufsverläufen fördernden oder hemmenden Bedingungen unterliegen.

Für die Unternehmen bedeutet dies, dass in nächster Zukunft verstärkte Investitionen in die Personalentwicklung und Weiterbildung der alternden Mitarbeiter notwendig werden. Eine Wissensbeschaffung über die Rekrutierung Junger wird nicht mehr ausreichen. Es zeigt sich jedoch, dass sich unterschiedliche Qualifikationsprofile und Erfahrungen jüngerer und älterer Mitarbeiter ausgesprochen sinnvoll ergänzen können.

\subsection{Der Kunde bestimmt das Produkt}

Der Kunde von heute fordert Produkte, die genau auf seine Bedürfnisse zugeschnitten sind. Dabei handelt es sich nicht nur Produkte des täglichen Bedarfs, sondern auch um Produkte, die Langzeitcharakter haben. Dazu zählt sicherlich das 
Auto, das inzwischen als Vorzeigeprodukt einer ausgeprägt kundenorientierten Branche gilt. Um dieses Produkt herum werden immer mehr Dienstleistungen angeboten, die ebenfalls individuell ausgestaltet sind. In den Unternehmen hat diese Entwicklung einen deutlichen Einfluss auf die Gestaltung der Produktions- und Geschäftsprozesse in Richtung einer nachhaltigen Flexibilität gehabt. Flexibilisierung kann aber nur erreicht werden, wenn sie mit einer individuellen Mitarbeiterqualifizierung einhergeht.

\subsection{Flexibilisierung in der Zusammenarbeit}

Die Maxime "Arbeite in einer zentralen Struktur, am fixen Ort und zur festen Zeit" wird abgelöst von einer zeitlichen, räumlichen und strukturellen Mobilität nach dem Motto: Arbeite mit wem, wann und wo du willst! Vorreiter dieser Entwicklung war die Flexibilisierung der Arbeitszeit, die mit der Einführung der Gleitzeit begann. Hinzu kam Anfang der neunziger Jahre die räumliche Mobilität. Das Arbeiten zu Hause, beim Kunden oder unterwegs gehört für viele schon jetzt zum Alltag. Dazu kam die Auflösung starrer Unternehmensstrukturen hin zum virtuellen Unternehmen, einem temporären Netzwerk unabhängiger Firmen, die mittels moderner Informations- und Kommunikationstechniken eine Aufgabenstellung gemeinsam bearbeiten. Auf die Industrie- und Büro-Baustrukturen wird sich dieser Trend zur Flexibilisierung genauso auswirken wie auf den privaten Wohnungsbau.

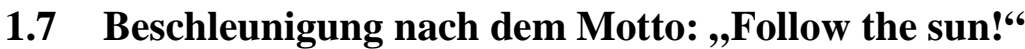

$\mathrm{Zu}$ den Trends Global Sourcing und Global Manufacturing kommt immer mehr der Trend Global Engineering. Rund um die Uhr und rund um die Welt wird nach dem Motto „Follow the sun“ in virtuellen, verteilten Teams ein neues Produkt entwickelt. Der Einsatz moderner Informations- und Kommunikationstechnologien mit großen Bandbreiten ermöglicht die Übermittlung komplexer und großer Datenmengen wie sie z.B. bei CAD-Entwicklungen anfallen. Mit dieser Art der Unternehmensvernetzung ist eine rasante Beschleunigung des Entwicklungsprozesses verbunden. Damit einher geht eine Beschleunigung des Informations- und Wissenstransfers und führt zu einem Wissenswettbewerb der Unternehmen auf dieser Welt.

\section{Wissen und Wissensvorsprung}

Für Unternehmen wird es immer schwieriger, exklusives Wissen aufzubauen und einen substantiellen Wissensvorsprung zu halten. Deshalb ist selbst in großen, 
weltweit operierenden Konzernen eine Konzentration auf Kernkompetenzen zu erkennen. Diese Spezialisierung erleichtert die Entwicklung innovativer Produkte. Es wird in Zukunft für viele Unternehmen zunehmend schwieriger, ein breites, umfassendes Leistungsspektrum anzubieten. Daraus resultiert die Notwendigkeit zu Zusammenschlüssen in Unternehmensverbünden, wie beispielsweise zu virtuellen Unternehmen oder Kompetenznetzwerken.

Als Konsequenz dieser Entwicklungen stellt sich die Frage, welche Fähigkeiten die Gesellschaft und die Unternehmen zukünftig entwickeln müssen. Die hohen Fortschrittsgeschwindigkeiten in der Wissenschaft und der Industrie fordern die Menschen in zunehmenden Maße. Die individuelle, kundenorientierte Lösungsentwicklung erfordert neben einer ausgeprägten Problemlösungskompetenz, das Verständnis von Arbeitsabläufen, -strukturen und Problemstellungen des Kunden. Die Fähigkeit zur schnellen Erschließung neuer Wissensgebiete und die Fähigkeit zur multidisziplinären Zusammenarbeit werden damit zu den Schlüsselkompetenzen eines Mitarbeiters.

Die logische Folge ist eine Gesellschaft, welche das lebenslange und vor allem das bedarfsgerechte Lernen in den Mittelpunkt stellen muss. Für die Unternehmen in der Wissensgesellschaft stellt sich die Frage, wie zukünftig die vorhandene Ressource Wissen effizient genutzt, weiterentwickelt und dem Kunden bzw. dem Kooperationspartner bedarfsgerecht zur Verfügung gestellt werden kann. Wissen wird zur strategischen Ressource im Produktionsprozess, im Produkt und es wird selbst zum Produkt. Ein erfolgreiches Management dieses Wissens erfordert aber einen gewissen technologischen, organisatorischen und unternehmenskulturellen Reifegrad des Unternehmens, der die Voraussetzung ist für die Möglichkeit der Wissensvernetzung im Unternehmen und für die Umsetzung des Wissens in Produkte. Die Fähigkeit zur effizienten Nutzung und Weiterentwicklung des eigenen Wissens ist aber auch eine Voraussetzung, um erfolgreich in wissensintensiven Kooperationen agieren zu können. Die Unternehmen, die es schaffen, ihr Wissen nach Bedarf zu bündeln und sowohl inner- als auch zwischenbetrieblich optimal zu nutzen und bereitzustellen, werden den Markt der wissensintensiven Güter und Dienstleistungen beherrschen.

\section{Innovationen fördern - Zukunft gestalten}

Der Mensch neigt dazu, Veränderungen gegenüber eher skeptisch zu sein. Veränderungen sind mit Unsicherheit verbunden und können nie genau vorhergesagt werden. Häufig werden sie bekämpft, ehe sie angenommen, gestaltet und für selbstverständlich gehalten werden. Dies gilt sowohl für neue Produkte als auch für Prozess- und Strukturveränderungen. In wirtschaftlich schwierigen Zeiten werden Veränderungen eher als Chance anstatt als Risiko betrachtet. Trotzdem 
erfolgt solch ein Wandel nicht automatisch. Er muss immer wieder angepasst werden und dies erfordert Innovationen auf allen Gebieten.

Wenn Innovation gelingt, erscheint das oft als Kunststück. Es ist aber eine Kunst, die man erlernen kann. Neben Phantasie und Mut gehört auch Können dazu, neue Ideen erfolgreich umzusetzen. Und hier sind nicht nur die Unternehmen sondern vor allem alle Bildungseinrichtungen gefordert.

Auf der einen Seite geht es um die Vermittlung von Fachkompetenz. Dazu gehört neben dem fachlichen Grundlagen- und Spezialwissen auch ein interdisziplinäres und interkulturelles Wissen. Des Weiteren ist Methodenkompetenz gefordert, mit der Probleme gelöst und Entscheidungen gefällt werden. Auch die Fähigkeit, Fachwissen zu kombinieren und zu ergänzen spielt eine wesentliche Rolle im Innovationsprozess. Auf der anderen Seite steht die Sozialkompetenz: die Fähigkeit im Team zu arbeiten und sich selbst zu motivieren sind hier entscheidende Aspekte.

Notwendig wird eine in hohem Maße auf Vertrauen basierende Unternehmenskultur mit Führungskräften und Mitarbeitern, die ein gemeinsames Zielsystem vereinbaren, das aber auch ausreichende Gestaltungsmöglichkeiten für den Einzelnen bietet. Nur mit einer wirklich von allen gelebten Vertrauenskultur können die Herausforderungen einer flexiblen Arbeitsorganisation für Unternehmen und Mitarbeiter zum Vorteil gereichen. Was wir dazu benötigen, sind innovative und kreative Menschen, die bereit sind, die vielfältigen Aufgaben in unserer Gesellschaft anzugehen. 